\title{
L'As du Lycée, une contribution à la connaissance des droits de l'enfant congolais ? La réception de la série télévisée dans le milieu scolaire kinois.
}

\author{
Arlette Masamuna Silumvumina \\ Université Catholique du Congo
}

\section{Introduction}

Les recherches sur les séries télévisées, individuelles ou collectives, attirent de plus en plus l'attention des professionnels, experts et scientifiques. Cependant, l'on observe que la plupart de ces études sont consacrées aux séries américaines ${ }^{1}$. En République Démocratique du Congo, les études de ce genre ne sont pas encore très développées. Elles sont généralement cantonnées aux mémoires d'étudiants et demeurent inédites. De quoi affirmer qu'une réflexion consacrée aux séries télévisées est novatrice dans la tradition de recherches congolaises. Cette situation est d'autant plus intéressante que le pays a récemment accueilli les travaux du $\mathrm{XIV}^{\mathrm{e}}$ sommet de la Francophonie à Kinshasa du 12 au 14 octobre $2012^{2}$.

Notre étude se focalise sur l'analyse d'une série francophone ${ }^{3}$ qui a pour titre $L ' A s d u$ Lycée. Cette fiction est produite au Burkina Faso en Afrique de l'Ouest. Le programme est diffusé dans l'espace télévisuel congolais à travers deux chaînes, à savoir CEBS et Rtnc2 ${ }^{4}$.

Les personnages de la série étant en majorité des enfants ${ }^{5}$ et les intrigues mettant en exergue les droits de ces derniers, le présent article se focalisera sur le type du discours véhiculé par la fiction sur ce sujet et, dans un deuxième temps, sur ce qu'en comprennent les enfants qui regardent le programme. Ce texte articule donc deux méthodologies : une analyse de contenu et une étude de réception. Mais avant de développer ces parties, il nous semble important de rappeler le contexte législatif congolais relatif

\footnotetext{
${ }^{1}$ Nous renvoyons, par exemple, à l'ouvrage de Sarah Sepulchre (Décoder les séries télévisées, 2011) où la plupart des séries étudiées et citées comme référence sont américaines, et notamment les travaux précurseurs de E. Katz et T. Liebes (The Export of Meaning, 1990) où ils se sont intéressés à la perception et l'interprétation du feuilleton américain Dallas.

${ }^{2}$ L'enquête de terrain liée à cet article a été réalisé en 2013.

${ }^{3}$ L'épithète francophone renvoie à la langue française (le français) mais aussi au partage du français.

${ }^{4}$ La première est une chaîne privée confessionnelle tandis que la seconde est une chaîne publique.

${ }^{5}$ Dans la loi no 09/001 du 10 janvier 2009 portant protection de l'enfant en République Démocratique du Congo, on entend par "enfant", toute personne âgée de moins de dixhuit ans (art.2, alinéa 1).
} 
aux droits des enfants d'une part et d'aborder rapidement le champ de l'éducation par les médias d'autre part.

\section{Les droits de l'enfant en République Démocratique du Congo}

La protection des droits de l'enfant congolais est exprimée dans la Constitution de la République Démocratique du Congo de 2006. Les applications de ce droit sont inscrites dans la loi 09/001 du 10 janvier 2009 à l'article $1^{\text {er }}$ : «La présente loi détermine les principes fondamentaux relatifs à la protection et à la promotion des droits de l'enfant conformément aux articles 122, point 5,123, point 16 et 149 alinéa 5 de la Constitution ».

Dans l'exposé des motifs de ladite loi, le législateur s'attarde sur les objectifs poursuivis, notamment « diffuser et promouvoir la culture des droits et devoirs de l'enfant et d'en faire connaître à celui-ci des particularités intrinsèques en vue de garantir l'épanouissement intégral de sa personnalité et de le préparer à ses responsabilités citoyennes ; cultiver en lui les valeurs de solidarité, de tolérance, de paix et de respect mutuel afin de l'amener à prendre conscience de l'indissociabilité de ses droits et devoirs par rapport à ceux du reste de la communauté ».

La loi congolaise prévoit donc, non seulement, la protection des droits de l'enfant, mais également une éducation à ces matières. Depuis 2010, le Ministère du Genre, Famille et Enfant s'emploie à la vulgarisation et à la sensibilisation aux droits de l'enfant avec le concours de certaines missions diplomatiques accréditées ou avec l'appui de certaines agences des Nations-Unies en République Démocratique du Congo. Ceci s'opère à travers des campagnes médiatiques (spots ou saynètes) mettant en scène surtout les artistes comédiens ou musiciens les plus en vue. Les enfants eux-mêmes apparaissent rarement dans ces programmes. La langue utilisée dans ces campagnes est généralement le Lingala. À défaut, on remarque aussi l'apparition des autres langues nationales, Ciluba, Kikongo et Swahili, alors que le français n'est quasiment jamais employé 6

Un exemple manifeste de l'attention portée à ces thématiques est l'accent mis sur les problématiques d'éducation aux médias par l'Université Catholique du Congo (anciennes Facultés Catholiques de Kinshasa) durant la célébration de la $41^{\mathrm{e}}$ Journée Mondiale des Communications Sociales. À cette occasion, l'objectif des activités commémoratives a été rappelé. Cette journée visait à :

\footnotetext{
${ }^{6} \mathrm{Il}$ n'existe malheureusement pas d'études systématiques sur les langues utilisées dans la production congolaise. Il est donc impossible d'établir un panorama précis sur cette question. On peut cependant préciser que la plupart des fictions en français proviennent de l'extérieur (les chaînes africaines, notamment TV5 Afrique) à côté d'une infime part produite dans le pays. Par contre, les programmes en langues locales sont créés sur place.
} 
[...] contribuer à la promotion de l'éducation de la jeunesse par les médias et à en faire une priorité sociale de leurs actions par une mobilisation permanente des acteurs médiatiques, politiques et scientifiques. Une telle ambition vise à renforcer, par l'information et la formation, les capacités des professionnels des médias en les rendant proactifs dans la médiatisation des savoirs scientifiques à destination des enfants (Budim'Bani, « Note de présentation »).

Cette rencontre qui a réuni les scientifiques, les politiques et les responsables de programmation des médias de Kinshasa était, pour les organisateurs de ces assises, une occasion de s'interroger sur la place que les médias accordent aux enfants et aux jeunes dans les programmes éducatifs et de divertissement.

En effet, la loi congolaise impose aux médias de protéger l'enfant de toute exposition à ce qui est nocif, troublant et violent. Les autorités du pays encouragent également la diffusion de programmes contribuant à l'épanouissement de ce public. Toussaint Tshilombo Send, alors ministre de l'Information, Presse et Communication Nationale, écrit:

C'est pourquoi, la réforme du paysage médiatique congolais que j’ai décidé conformément à la décision du Conseil des ministres du 24 mars dernier, la réglementation en cette matière va être renforcée afin que la révision des grilles des programmes et des cahiers des charges telle qu'envisagée puisse accorder une part significative aux émissions dédiées à l'enfant tant en ce qui concerne son information que son divertissement (Tshilombo Send, "Allocution de S.E. Monsieur le Ministre de l'Information, Presse et Communication nationale »).

Au regard de ce qui précède, la diffusion de la série L'As du Lycée pourrait être considérée comme un moyen utilisé par les chaînes et les diffuseurs pour se conformer à leur cahier des charges en offrant aux plus jeunes des programmes attrayants qui concourent à leur formation, particulièrement concernant leurs droits. Cependant, avant de vérifier si les enfants qui regardent ce programme intègrent effectivement des savoirs juridiques, il est nécessaire de présenter les bases de l'éducation non formelle.

\section{Apprendre par les séries télévisées}

À la suite de la théorie de l'adoption d'Everett Rogers (1962), nous considérons que les médias ont la capacité de changer les mentalités. En 1973, Rogers et F. Floyd Schoemaker ont conçu un modèle d'adoption des innovations techniques. Ce modèle, écrit Gilles Willet, «a ensuite été appliqué aux pays du Tiers-Monde et ensuite dans d'autres domaines tels que la santé, l'éducation, la vie politique » (414). Everett Rogers conçoit que les médias peuvent être utilisés afin d'inculquer certains savoirs à 
leurs usagers. Cette théorie se rapproche du paradigme des savoirs informels/non formels des spécialistes de l'éducation ${ }^{7}$.

Pour Daniel Jacobi le terme de l'éducation non formelle se définit par opposition avec celui d'éducation formelle. Pour lui,

[...] l'éducation formelle désigne donc toutes les interventions à caractère éducatif qui sont mises en œuvre par des institutions ou des acteurs autres que l'appareil scolaire officiel. Autrement dit, la notion d'éducation non formelle ne peut être définie précisément, ou mieux construite, que par contraste avec le déroulement des apprentissages visant l'appropriation des savoirs formels, c'est-à-dire des savoirs scolaires (171).

Dans une perspective proche, Jack Guichard considère que le type d'apprentissage véhiculé par les médias est informel parce qu'il diffère des normes de l'école (205). Il souligne, à propos de la distinction entre les savoirs formels et informels que « la différenciation entre savoirs formels et informels passe par l'analyse des différences entre les domaines scolaire et médiatique » (203).

Nous émettons donc l'hypothèse que la série L'As du Lycée est un support médiatique pouvant initier des changements dans la connaissance de leurs droits chez les enfants congolais et qu'elle produit, à ce titre, des savoirs informels. Nous vérifierons ce postulat par une étude de réception auprès de jeunes filles congolaises. Dans un premier temps, la partie empirique de cet article s'ouvre sur une présentation de la série et une étude de son contenu.

\section{L'As du Lycée, les droits des adolescents}

L'As du Lycée a été créée en 2008 par une réalisatrice burkinabè, Missa Hebie. Une année après sa sortie, ce programme a remporté le prix de la meilleure série TV/Vidéo et le prix des Nations Unies Droits des enfants (Unicef/Burkina) au Festival Panafricain du Cinéma de Ouadougou, Fespaco. La fiction a également été récompensée du prix Africa numérique au Festival Vues d'Afrique de Montréal en 2009.

L'As du Lycée raconte l'histoire d'Ismaël, un enfant de 12 ans, d'origines modestes (son père est concierge). Il présente de sérieuses capacités intellectuelles, mais ses parents n'ont pas les moyens d'assurer son instruction. Le patron de son père, Monsieur Pascal, décide d'inscrire Ismaël dans le même lycée que sa propre fille, Fanny. Malgré l'amitié de cette dernière, l'intégration d'Ismaël dans cet établissement réservé aux enfants nés dans des milieux aisés est complexe. Ismaël n'est pas le bienvenu dans l'école, ni dans les endroits que fréquentent ses camarades de classe. Certains sites touristiques, d'autres lieux de loisirs et même

${ }^{7}$ Pour en savoir plus, nous renvoyons les lecteurs aux numéros 15 et 16 de 2001 de la revue Recherches en Communication. 
l'église (catholique) située en ville lui sont interdits. Fanny, qui lui est très attachée, lui sert de mentor dans toutes les situations et tente de favoriser son intégration.

\subsection{Analyse de contenu : présentation méthodologique}

Notre corpus est composé des trois épisodes les plus cités par les élèves interrogées ${ }^{8}$ durant l'étude de réception. Ils sont issus de la saison 51-100 et durent chacun 15 minutes. Il s'agit de La rentrée de classe, Le suspect $n^{\circ} 1$ et L'anniversaire. Étant donné que la fiction est peu connue en dehors du continent africain, quelques mots concernant les histoires sélectionnées ne semblent pas superflus.

Le premier épisode, La rentrée de classe, raconte le premier jour d'Ismaël dans son nouveau lycée. Ismaël doit faire face aux réticences des adultes, particulièrement la maman de Fanny qui n’apprécie pas sa présence dans l'école, et des autres élèves. Durant l'épisode Suspect $n^{\circ} 1$, Ismaël est accusé du vol d'un téléphone portable. Tous les personnages, à part Fanny et son propre père, sont convaincus qu'il est coupable. Ils devront prouver l'innocence d'Ismaël. Enfin, dans L'anniversaire, Fanny souhaite inviter Ismaël à sa fête, ce que sa mère refuse catégoriquement. Au milieu de la célébration, Ismaël apparait en costume ethnique traditionnel.

L'analyse de contenu proposée est qualitative et non quantitative. Notre intérêt, dans ce travail, consiste à dégager les idées majeures en rapport avec les droits de l'enfant qui émanent du récit filmique. Nous allons procéder en deux temps : une analyse narratologique basée sur le schéma actanciel d'Algirdas Julien Greimas permettant de mettre en évidence les forces en présence dans le texte et une analyse de discours permettant de faire émerger les propos les plus saillants concernant les droits des enfants.

Comme il est question d'analyser un texte narratif télévisuel, la notion du récit (médiatique) demeure un concept clé. Les spécialistes, quel que soit leur domaine de prédilection, considèrent que tout récit commence par un manque. Marc Lits souligne: «Toute narration repose sur un déséquilibre initial qui doit être comblé » (145). Le schéma narratif de L'As du Lycée obéit bel et bien à cette logique. Ceci est d'autant plus crucial que l'intrigue des épisodes sélectionnés se concentre justement sur les droits d'Ismaël ce qui va nous permettre d'étudier comment ce thème est présenté par la série.

Greimas propose d'étudier les fictions à partir des rôles importants incarnés par les personnages qu'il modélise dans un schéma actanciel. Selon le théoricien, «toute histoire se construit selon une logique

${ }^{8}$ Nous reviendrons dans la seconde partie de l'article sur les propos des élèves interrogées. 
d'enchaînements de séquences narratives, et autour de quelques figures actancielles qui organisent le récit» (Lits 144). Pour cette analyse, nous nous attarderons principalement sur l'axe du pouvoir. "L'axe du pouvoir met en scène l'adjuvant et l'opposant. L'adjuvant aide le sujet à atteindre son objet, tandis que l'opposant lui fait obstacle. Un anti-sujet est bien sûr un opposant, mais tout opposant n'est pas un anti-sujet» (Lits 68). La fiction étant centrée sur les droits d'Ismaël, c'est donc autour de cette thématique que les rapports de pouvoir vont probablement se concentrer.

L'analyse de discours sera constituée par une analyse des thèmes présents dans les épisodes et des dialogues des personnages. Le thème des droits de l'enfant n'est pas énoncé tel quel dans la fiction, mais il apparait au travers des différents sujets traités (la culpabilité, l'intégration ou le rejet, le droit à l'éducation, etc.,) et par les prises de position des différents personnages. Leur examen systématique va permettre de comprendre les valeurs véhiculées par la série.

\subsection{Résultats}

La présentation des résultats se fera en deux étapes. Dans une première phase, les actants, les personnages et leur discours seront listés dans un tableau. Dans un second temps, les thèmes développés par le récit seront examinés.

Tableau $\mathrm{n}^{\circ} 1$ : Représentation de l'axe du pouvoir de $L^{\prime} A s d u$ lycée.

\begin{tabular}{|c|c|c|}
\hline $\begin{array}{l}\text { Axe du } \\
\text { pouvoir }\end{array}$ & Personnages & Discours et actions \\
\hline \multirow[t]{3}{*}{ Opposants } & Mère de Fanny & $\begin{array}{l}\text { «Il n'est pas de notre milieu » }\left(1^{9}\right) \\
\text { Elle accepte qu'on fouille la maison } \\
\text { d'Ismaël pour trouver le téléphone (2) } \\
\text { Elle refuse d'inviter Ismaël à la fête de } \\
\text { Fanny (3) } \\
\text { Elle refuse qu'on serve la famille } \\
\text { d'Ismaël lors de la fête (3) } \\
\text { "Je ne vois pas les noms de Djoe et } \\
\text { de Patricia sur la liste des invités ? " } \\
\text { (3) }\end{array}$ \\
\hline & Patricia & $\begin{array}{l}\text { Elle est étonnée de voir Ismaël dans } \\
\text { son école (1) } \\
\text { Elle vole le téléphone (2) }\end{array}$ \\
\hline & Les collègues de & Ils se moquent (1) \\
\hline
\end{tabular}

${ }^{9}$ Les numéros renvoient aux trois épisodes dont les éléments sont tirés : $1=$ La rentrée des classes, $2=$ Suspect $^{\circ} 1,3=$ L'anniversaire. $^{\circ}$ 


\begin{tabular}{|c|c|c|}
\hline & \multicolumn{2}{|l|}{ classe } \\
\hline & Djoe & $\begin{array}{l}\text { Il traite Ismaël de « macaque » (1) } \\
\text { Il teste Ismaël (1) } \\
\text { Il suspecte Ismaël de vol (2) } \\
\end{array}$ \\
\hline & Le proviseur & $\begin{array}{l}\text { Il prend des sanctions plus fortes à } \\
\text { l'encontre d'Ismaël (1) } \\
\text { Il est à la tête de la délégation qui doit } \\
\text { fouiller la maison d'Ismaël (2) }\end{array}$ \\
\hline & Les autorités & $\begin{array}{l}\text { Elles sont convaincues qu'Ismaël est } \\
\text { coupable (2) } \\
\text { Elles finissent par accepter Ismaël (2) }\end{array}$ \\
\hline & Certains parents & $\begin{array}{l}\text { Ils sont convaincus qu'Ismaël n'est } \\
\text { pas à sa place (3) }\end{array}$ \\
\hline Adjuvants & Père de Fanny & $\begin{array}{l}\text { Il accepte qu'Ismaël aille à l'école de } \\
\text { Fanny (1) } \\
\text { Il se rend à l'école afin de défendre } \\
\text { Ismaël contre les fausses accusations } \\
\text { de Djoe (2) } \\
\text { Il accepte qu'il soit présent à } \\
\text { l'anniversaire (3) }\end{array}$ \\
\hline & Père d'Ismaël & $\begin{array}{l}\text { Il défend son fils (2) } \\
\text { Il admet qu'Ismaël puisse être à sa } \\
\text { place à la fête (3) }\end{array}$ \\
\hline & Fanny & $\begin{array}{l}\text { Elle demande pour qu'Ismaël puisse } \\
\text { venir à son école (1) } \\
\text { Elle présente Ismaël aux autres élèves } \\
(1) \\
\text { Elle défend Ismaël vis-à-vis de la } \\
\text { classe et des autorités (1) } \\
\text { Elle place Ismaël en tête sur la liste de } \\
\text { ses invités (3) }\end{array}$ \\
\hline & Ismaël & $\begin{array}{l}\text { Sa culture (lui permet de réussir le } \\
\text { test) (1) } \\
\text { Son courage (il confronte le fou) (1) } \\
\text { Sa ténacité (il clame son innocence) } \\
(2) \\
\text { Sa volonté (il travaille pour offrir un } \\
\text { cadeau à Fanny) (3) } \\
\text { Son costume traditionnel (3) }\end{array}$ \\
\hline & $\begin{array}{l}\text { Les collègues de } \\
\text { classe }\end{array}$ & $\begin{array}{l}\text { Ils se moquent de Djoe (1) } \\
\text { Ils applaudissent }\end{array}$ \\
\hline & Notiandi & Elle aide Ismaël à entrer à la fête (3) \\
\hline & $\begin{array}{l}\text { Le père d'un } \\
\text { invité à la fête }\end{array}$ & $\begin{array}{l}\text { Il est impressionné par le costume } \\
\text { d'Ismaël et le complimente (3) }\end{array}$ \\
\hline
\end{tabular}




\begin{tabular}{|l|l|l|}
\hline & Les invités & Ils applaudissent Ismaël (3) \\
\hline Sujet (héros) & Ismaël & Défense de ses droits \\
\hline
\end{tabular}

Ismaël est donc le sujet en quête d'un objet (le respect de ses droits). Dans son parcours, il rencontre des personnages qui l'aident à atteindre son but, les adjuvants : le père de Fanny, Fanny, son propre père, certains amis de la classe, etc. Des opposants tentent d'empêcher son intégration : la mère de Fanny, le proviseur, Djoe, Patricia, etc. Le nombre d'adjuvants et d'opposant est similaire, respectivement sept et huit. On remarque également qu'il s'agit autant de personnages individualisés (Djoe, le proviseur) que de collectifs (les autorités, les invités).

Les adjuvants sont généralement des personnages "facilitateurs". Par exemple, Fanny guide Ismaël dans sa nouvelle école et Notiandi lui permet d'entrer à la fête. Ces personnages soulignent le fait que le héros est un enfant comme les autres («Laissez Ismaël, il est élève comme tout le monde »). D'autres ont une position dominante dans la société et parviennent à faire accepter Ismaël. C'est le cas du père d'un invité qui remarque la tenue traditionnelle d'Ismaël (« Madame, mais il est beau dans sa tenue traditionnelle ») ou du papa de Fanny qui parvient à l'inscrire dans l'établissement scolaire.

Les propos des opposants sont généralement excluants. Ils utilisent des arguments de classe ( $\mathrm{Il}$ n'est pas de notre milieu»), racistes ("macaque »), des préjugés (les autorités sont convaincues qu'il est coupable sans enquêter). Certaines personnes vont jusqu'à tenter de piéger Ismaël (c'est le cas de Patricia qui tente de le faire accuser de vol). Contrairement à ce qu'on pourrait croire, tous les riches ne sont pas des opposants (notamment Fanny et son père sont du côté d'Ismaël) et tous les pauvres ne sont pas adjuvants. En effet, les autorités du lycée, qui sont pourtant de condition modeste, comme le proviseur, rejettent le héros, peut-être pour plaire aux mieux nantis (le proviseur veut plaire à la maman de Djoe).

Malgré les obstacles rencontrés, fruit des actions des opposants, Ismaël persévère jusqu'à en sortir victorieux : on l'applaudit (La rentrée des classes), Patricia est démasquée pour le vol du téléphone (Le suspect $n^{\circ} 1$ ) et enfin il peut participer à la fête (L'anniversaire). Cependant, le plus important dans le processus narratif n'est pas tant cette victoire que le fait qu'il parvienne à se faire accepter, autrement dit que certains opposants changent d'avis et se rangent du côté des adjuvants. C'est le cas, par exemple, de certains collègues de classe ou du proviseur. Cet élément est loin d'être anodin. En effet, Lits souligne que, peu importe les grilles d'usage dans l'analyse du personnage, «c'est toujours le devenir des personnages qui constitue le fil directeur des actions et suppose la 
transformation des contenus»(Lits 139). Le changement d'opinion se produit sous l'influence de deux facteurs : les qualités d'Ismaël lui-même (sa culture, sa ténacité, son costume traditionnel, etc.) ainsi que les attitudes et discours des adjuvants (qui parviennent à convaincre les autres). C'est en cela que la série se fait éducatrice, comme les anciens opposants, les téléspectateurs sont amenés à réévaluer leurs positionnements et à changer de mentalité sous l'influence des arguments présentés par la fiction.

La transformation ne se produit que du côté des opposants. En effet, les adjuvants restent fidèles à leur positionnement initial qui consiste à prêter main-forte au héros pour qu'il puisse s'intégrer. L'insertion d'Ismaël dans l'école ne produit donc pas de contre-performance et reste totalement un plaidoyer pour la tolérance.

Quant aux thèmes développés dans cette série, on peut affirmer que le principal est le droit de l'enfant. Cependant, dans le récit, ce topic n'est pas explicite. La série utilise des personnages individualisés, des situations concrètes, des actions particulières, des dialogues précis qui illustrent certains aspects de cette thématique. Tous ces éléments forment en définitive un discours contre l'exclusion sociale, pour le rétablissement de la justice, pour l'éducation de chaque enfant, pour le respect et la considération de tous, etc. Mais ce discours reste majoritairement implicite.

À certains moments, toutefois, des discours font référence plus directement aux droits des enfants et, notamment, à l'égalité de tous, l'intégration sociale, le droit à l'éducation pour tous, le droit à la justice, la dignité pour tous. Cependant, le lien avec la loi n'est jamais établi.

\section{L'As du Lycée, ce que les adolescents en pensent}

Nous venons de constater que les intrigues des épisodes mettent effectivement en avant des éléments liés aux droits des enfants congolais. Cependant, cela ne signifie pas nécessairement que ces propos soient repérés, compris et intégrés par les spectateurs. Dans une deuxième partie de la recherche, nous avons donc souhaité rencontrer des adolescents afin de vérifier ce qu'ils retiennent de la série.

\section{1. Étude de réception, présentation méthodologique}

Les études liées à la réception se situent dans le champ théorique qui s'intéresse aux effets des médias. Trois grandes périodes ont jalonné ces recherches : durant la première, les chercheurs pensent que les médias sont tout-puissants et produisent des conséquences directes sur les récepteurs; durant la seconde ère, les recherches établissent que les médias engendrent des effets limités sur leurs lecteurs; le troisième moment prouve que la réception est un phénomène complexe (Cabin 293-296). Aujourd'hui, ce champ rassemble des recherches très 
différentes portant sur des objets très divers, des thématiques variées; cette situation est liée également à l'évolution du paysage médiatique. Dans la perspective de la reconnaissance du foisonnement des travaux découlant de ce champ, Laurence Doury précise que «certains travaux (sur la réception) privilégient l'analyse du contenu ou de la structure de l'objet, tandis que d'autres interrogent les individus sur leurs rapports à l'objet » (218). Dans cet article, nous nous concentrons sur le premier axe, l'analyse de contenu, en nous intéressant à ce que le public retient des histoires développées dans la fiction.

La présente étude sur la réception de la sérié télévisée $L ' A s$ du Lycée s'est déroulée en République Démocratique du Congo dans un lycée pour filles, le Lycée Boyokani. Cet établissement d'enseignement secondaire est situé au centre de la capitale congolaise, Kinshasa, à Yolo-Sud dans la commune de Kalamu non loin d'un autre quartier de la même commune mais très connu, le quartier "Matonge ». Cette école fait partie de l'enseignement confessionnel catholique qui se trouve, sur le plan ecclésiastique, dans la Région apostolique de Kin-Centre (Etsou 32). Ce lycée reçoit des enfants de toutes les couches sociales (autant des enfants de cadres que d'ouvriers, de gouvernants que de gouvernés, de riches ou de pauvres). Cette caractéristique de mixité nous a paru être une richesse pour la recherche menée.

Pour analyser les usages des téléspectateurs, Doury renseigne que les chercheurs recourent principalement à trois techniques : les observations de situation de consommations des médias, les entretiens avec les personnes à propos de leur activité télévisuelle et les questionnaires (158). Nous avons réalisé une étude de terrain qualitative à l'aide d'entretiens collectifs. Cinquante étudiantes entre 12 et 14 ans ont été rencontrées par groupe de dix personnes. Ces groupes témoins ont été entendus au mois de décembre 2013. À cause de certaines influences et interférences observées $^{10}$, ces entretiens collectifs ont été complétés par des entrevues individuelles sur un effectif de dix élèves. Au sein de chaque groupe, deux élèves ont été sélectionnées pour cette deuxième phase qui a été organisée durant la même période. Ce sont ces entretiens individuels qui seront traités dans la suite de cet article.

Durant les entretiens, nous avons demandé aux élèves de raconter l'histoire de la série et de certains épisodes particuliers ${ }^{11}$. À partir du discours recueilli, nous avons centré nos questions sur le traitement

\footnotetext{
10 Nous avons remarqué que certaines élèves se laissaient influencer par d'autres, principalement leurs amies, et changeaient leur propos. D’autres ont été désorientées par les rires de leurs pairs.

${ }^{11}$ Les trois épisodes les plus racontés par les lycéennes, La rentrée des classes, Le suspect n ${ }^{\circ} 1$ et L'anniversaire, constituent l'échantillon sur lequel se base notre réflexion.
} 
infligé au héros de l'histoire en faisant le rapprochement avec les droits de l'enfant afin de vérifier les valeurs perçues par les élèves interrogées.

\subsection{Résultats}

Tout d'abord, il convient de préciser que les élèves portent un grand intérêt à la série. Elles sont véritablement passionnées par l'histoire et elles sont capables de la restituer de manière précise durant les entretiens. On peut donc affirmer, à la suite de Jack Guichard, que les adolescentes se sont bien approprié la fiction (Guichard 207).

Le premier constat qu'il est permis de tirer des entretiens est que les filles interrogées sont capables de porter un jugement sur les actes des personnages. Les participantes ont repéré la discrimination, la stigmatisation et l'exclusion dont le héros fait l'objet. Particulièrement, toutes les intervenantes considèrent à l'unanimité que la méchanceté de la maman de Fanny est démesurée. Cette dernière refuse qu'Ismaël puisse étudier dans la même classe que sa fille Fanny aux frais de son mari. Deux faits sont jugés comme particulièrement ignobles : ordonner la fouille de la maison d'Ismaël (Le suspect $n^{\circ} 1$ ) et le refus catégorique de l'accepter à la fête de Fanny alors qu'ils habitent dans la même concession (L'anniversaire).

Elles sont également capables de relier ces histoires avec les situations de certains enfants congolais dans la réalité. Par exemple, dans la fiction, la maman de Fanny traite Ismaël de sorcier. Les adolescentes ont établi un rapprochement entre la victimisation d'Ismaël comme "enfant sorcier" avec la manière dont on traite les orphelins congolais condamnés à vivre dans la rue. Elles ont pu également établir un lien avec la discrimination vécue par Ismaël et leur propre vécu, même si elles ne sont pas elles-mêmes issues d'un milieu pauvre.

Cependant, elles ne font pas référence aux droits de l'enfant. Pour juger les comportements des personnages (principalement les opposants), elles en appellent à la morale ou à la justice divine comme instance de sanction. Certains actes sont perçus comme des manquements graves aux préceptes divins et à la morale. Le recours à la morale chrétienne et à la justice divine peut se justifier, d'une part, par l'identité chrétienne de l'établissement scolaire et, d'autre part, par l'hyper religiosité du milieu congolais en général et kinois en particulier (CERA 1983, Evêché de Matadi 1993). La prolifération des églises de réveils, l'organisation régulière de campagnes d'évangélisations fortement médiatisées, l'emploi d'édifices publics, par exemple des stades de football, pour des événements religieux sont probablement les manifestations les plus visibles de cette tendance. Cette conception va jusqu'à influencer le regard porté sur les personnages. Celles et ceux qui aident Ismaël sont perçus comme des saints. Par contre, ceux qui s'opposent à lui ou le haïssent sont qualifiés de «sorcier» ou de 
«méchant ». Le père de Fanny appartient clairement à la première catégorie tandis que la mère de Fanny se positionne dans la deuxième.

Les adolescentes perçoivent donc que ces comportements sont répréhensibles. C'est cette prise de conscience qui montre que la série concoure à la formation des adolescents puisque «les enfants en particulier ont des rapports aux objets et aux situations bien différents de ceux des adultes » (Guichard 209). Mais cela reste une connaissance tacite du droit, car elles n'évaluent jamais ces actes en termes légaux. Il apparait donc que les valeurs chrétiennes ou morales sont un référent mieux connu des adolescentes que le droit qu'elles ne mentionnent jamais spontanément ${ }^{12}$.

Enfin, nous avons voulu tester leur connaissance du droit de l'enfant par une question directe (qu'est-ce que le droit de l'enfant?). Certains ont répondu : «C'est une affaire des enfants d'Europe ». Ceux qui intégraient le concept à la vie des jeunes Congolais le traduisaient uniquement en termes de devoirs: "Les enfants doivent remplir leur devoir », "Les droits des enfants, signe de désobéissance aux parents ». Seule une poignée d'élèves ont évoqué les droits: "Les droits de l'enfant épanouissent l'enfant en société », " permettent aux enfants d'étudier », "permettent à l'enfant de s'exprimer ». La plupart des adolescentes conçoivent donc que les enfants ont surtout des devoirs (par exemple, vis-à-vis des parents). Cette situation est révélatrice d'un contexte où les jeunes baignent dans ce qui est appelé, au Congo, «la culture du devoir» (Elungu 85-86). Dans ce contexte, les fictions qui présentent cet aspect de la loi peuvent aider les adolescents à apprendre qu'ils ont aussi des droits.

\section{Conclusion}

Nous postulions, au début de cet article, que la série $L ' A s$ du lycée mettait en scène les droits des enfants et que la fiction pouvait aider les spectateurs à intégrer ces notions. Il s'agit d'un thème important pour le Congo qui a voté une loi en ce sens ( $\mathrm{n}^{\circ}$ 09/001 du 10 janvier 2009).

L'analyse de contenu nous a permis de constater que le thème principal de la série est bien le droit de l'enfant. Cette fiction véhicule donc des savoirs législatifs. Cependant, ils restent implicites dans le récit. Ils sont représentés par des personnages qui tentent de faire respecter leurs droits (le héros) ou celui des autres (les adjuvants). Les actes et les discours des protagonistes sont les éléments tangibles qui peuvent être repérés dans un récit, ils permettent d'incarner, d'exemplifier les thématiques. Cependant, il n'est pas certain que les spectateurs établissent un lien entre ces éléments et le droit des enfants.

\footnotetext{
${ }^{12}$ Sauf quand nous leur avons posé clairement la question sur ce que signifie les droits des enfants.
} 
Durant les entretiens, nous avons pu constater que les téléspectatrices ont en effet pu percevoir les discriminations dont le héros est victime. Elles ont également été capables de développer une réflexion autour de ces situations. Ces résultats confirment donc que les savoirs intégrés par les fictions sont perçus par les jeunes. Cependant, les adolescentes n’ont pas utilisé la loi comme référence à leurs raisonnements, mais bien la religion et la morale. Si elles ont remarqué les conflits présents dans le récit, elles n'ont pas pris conscience des éléments de droit sousentendus. Ce point nous amène à nuancer quelque peu notre hypothèse de départ. En effet, les savoirs sont repérés et peuvent être intégrés dans une réflexion, mais ils ne sont pas pleinement reconnus pour ce qu'ils sont. Ce qui reste implicite dans les récits n'est pas pleinement conceptualisé par les adolescentes. Cette observation pourrait constituer une piste d'amélioration des fictions qui tentent d'éduquer les publics (viser des programmes plus didactiques) ou la nécessité de produire des opportunités pour discuter de ces problématiques en distinguant la morale, la religion et la loi comme cette recherche le montre.

Ainsi, il est apparu que le processus de la recherche lui-même a eu un impact sur les savoirs des enfants. En effet, si au départ, les filles interrogées jugeaient les comportements des opposants en regard de la morale et de la religion, la discussion avec la chercheuse et les autres intervenantes leur a permis d'établir le lien avec le droit de l'enfant. Elles ont pris conscience que le droit réglemente les us et coutumes, condamne ce genre de comportements, voire les sanctionne. Elles ont compris que les droits de l'enfant sont illustrés dans la série par la mise en exergue de la valeur de l'égalité pour tous, du droit à la scolarité, etc.

On peut donc affirmer que L'As du Lycée a permis aux adolescentes de comprendre certains éléments, d'exercer leur capacité à juger des comportements, de les évaluer. La série constitue bien un outil de sensibilisation et de vulgarisation. Les fictions remplissent d'autant mieux cette fonction qu'elles ont du succès ( $c f$. l'appropriation dont les adolescentes étaient capables). Mais elle ne suffit pas à être une véritable instance médiatique d'apprentissage du droit des enfants. Un dialogue (ici avec la chercheuse) a été nécessaire afin que les personnes interrogées puissent établir le lien avec le droit des enfants.

Des recherches ont déjà établi le même genre de constat. Louis Porcher (1994) considère que la télévision et l'enseignement sont complémentaires. Si la télévision a des forces (elle peut contenir un nombre important de savoirs, elle est regardée dans des moments de divertissements, les histoires passent par des personnages avec lesquels on tisse des liens affectifs), elle possède aussi des faiblesses. Le théoricien prétend qu'on ne peut s'éduquer seul, notamment parce que nous ne sommes pas tous égaux face à la télévision et que nous devons apprendre à 
apprendre, ce qui est la fonction de l'école. Pierre Molinier souligne également que si la télévision peut faire passer des connaissances, ces éléments ne sont constitués en savoirs qu'à partir du moment où ils sont conscientisés, objectivés dans un discours et validés socialement (185-202). Or, les enfants, selon lui, ont du mal à objectiver les situations vues dans les médias sans un processus pédagogique d'accompagnement.

Il faudrait donc que les séries soient accompagnées de séances pédagogiques, de formations pour s'assurer que les enfants comprennent bien les notions en jeu dans les récits. La diffusion de fiction n'est pas suffisante, mais il s'agirait de mettre en place de véritables programmes d'éducation aux médias ou de sensibiliser les adultes qui encadrent les enfants (parents, éducateurs, professeurs), de compléter l'apprentissage informel par un apprentissage plus formel. 


\section{Bibliographie}

Budim'bani, François-Xavier. "Note de présentation." Médias et enfants. Actes de la $41^{e}$ Journée Mondiale des Communications Sociales. Éd. Facultés des Communications sociales, Facultés Catholiques de Kinshasa. Kinshasa : Logos, 2007.

Cabin, Philippe, ed. La communication. Etat des savoirs. Auxerre : Éd. Sciences Humaines, 1998.

Centre d'études des religions africaines (CERA). L'Afrique et ses formes de vie spirituelle. Actes du IIe colloque international du CER A, vol. 17, $\mathrm{n}^{\circ} 33-34$. Kinshasa : Faculté de Théologie Catholique de Kinshasa, 1983.

Doury, Laurence. "Comment analyser les publics des séries télévisées." Décoder les séries télévisées. Ed. Sarah Sepulchre. Bruxelles : De Boeck, 2011. 151-178.

Elungu, Pea. Tradition africaine et rationalité moderne. Paris : L'Harmattan, 1987.

Etsou, Frédéric, ed. Gouvernement pastoral de l'archidiocèse de Kinshasa. Kinshasa : Editions del'archidiocèse, 1993.

Evêché de Matadi. Religions et Développement social. Les sectes aujourd'bui. Actes du IIIe week-end moral des intellectuels chrétiens de Matadi. Matadi: Evêché de Matadi, 1993.

Guichard, Jack. "Prise en compte des rapports au savoir médiatique." Recherches en Communication 15 (2001) : 203-216.

Jacobi, Daniel. "Savoirs non formels ou apprentissages implicites?" Recherches en Communication 15 (2001) : 169-184.

Katz, Elihu and Tamar Liebes. The Export of Meaning : Cross-Cultural Readings of Dallas. New York: Oxford University Press, 1990.

Lits, Marc. Du récit au récit médiatique. Bruxelles : De Boeck, 2010.

Loi $\mathrm{n}^{\circ}$ 09/001 du 10 janvier 2009 portant protection de l'enfant en République Démocratique du Congo.

Molinier, Pierre. "Compréhension des médias et savoirs." Recherches en Communication 15 (2001) : 185-202.

Porcher, Louis. Télévision, culture, éducation. Paris : Armand Colin, 1994.

Rogers, Everett. Diffusion of innovations. New York: Free Press, 1962.

Sepulchre, Sarah, ed. Décoder les séries télévisées. Bruxelles : De Boeck, 2011.

Tshilombo Send, Toussaint. "Allocution de S.E. Monsieur le Ministre de l'Information, Presse et Communication nationale." Médias et enfants. Actes de la $41^{e}$ Journée Mondiale des Communications Sociales. Éd. Facultés des Communications sociales, Facultés Catholiques de Kinshasa. Kinshasa : Logos, 2007.

Willet, Gilles. La communication modélisée. Une introduction aux concepts, aux modèles et aux théories. Ottawa : Éditions du Renouveau Pédagogique, 1992. 